\title{
A sensitivity study of parameters used in shrinkage and creep prediction models
}

\author{
R. W. Howells, * R. J. Lark* and B. I. G. Barr* \\ Cardiff School of Engineering
}

Over the last 15 years, there have been numerous models put forward for the prediction of the time-dependent behaviour of concrete and consequently concrete structures. The development of these models from a number of different sources has meant that the engineer now has a choice to make when undertaking creep and shrinkage prediction and it is difficult to know which is best suited for this purpose. Some are considerably more complex than others requiring greater amounts of input focusing on material data, concrete properties, environmental conditions, specimen geometry and loading conditions. The study reported here focuses on the sensitivity to change, in both the short term and over time, of the individual parameters that comprise the input and whether one model is more appropriate in any given situation. It has been found that for each model certain parameters are more sensitive than others. It is suggested that for any given model, the parameters that do not reflect the expected behaviour when changed counterbalance each other, cancelling out any errors. This further suggests that when deciding on which model to use when predicting shrinkage and creep strains it is prudent to look at the specific conditions that prevail, assess the relevant input parameters for which data are available, assess the sensitivity level of each of these parameters and then make a decision as to the most appropriate model to use.

\section{Introduction}

Modern construction techniques enable concrete structures to be constructed rapidly. The loads generated during construction can be as large as the design service load. These construction loads can cause significant immediate deflections due to the low early-age modulus of the concrete and concrete cracking. Due to shrinkage and the significant initial stresses developed, the time-dependent deflections may be unacceptably large. Such situations warrant a review of the validity of current creep and shrinkage prediction methods.

Bažant and Baweja ${ }^{1}$ have identified that the realistic prediction of creep and shrinkage of concrete is a particularly difficult problem because the phenomenon is a result of several interacting physical mechanisms and is influenced by many variables. In view of this fact it is not surprising that improvements in prediction methods have evolved slowly and gradually. No obvious major breakthrough in the understanding of this

\footnotetext{
* Cardiff School of Engineering, Queen's Building, P.O. Box 925, Newport Road, CF24 OYF, Cardiff, UK.
}

(MCR 41249) Paper received 20 February 2004; last revised 14 June 2004; accepted 22 December 2004 phenomenon can be identified from the research literature. However, the accumulated advancement of knowledge since Ross ${ }^{2}$ first proposed a creep prediction chart in 1937, and especially during the last two decades, has been enormous. It is now possible to formulate a much better prediction model than 20 years ago.

Since 1990, there have been numerous prediction models put forward, generally by three main contributors; the American Concrete Institute (ACI), the Comité Euro-International du Béton (CEB), and Réunion Internationale des Laboratoires d'Essais et de recherche sur les Matériaux et les Constructions (RILEM). These models include the CEB-FIP Model Code 1990, ${ }^{3}$ the ACI Committee 209 model entitled 'Prediction of creep, shrinkage and temperature effects in concrete structures' from 1992, ${ }^{4}$ the BP-KX Model by Bažant et al. from 1991, ${ }^{5}$ the short-form of this model (BP-KX+) from 1993, ${ }^{6}$ the B3 Model by Bažant and Baweja from $1995^{1}$ and the short form of this model (B3+) from $1996 .^{7}$ In addition to these three main contributors, a number of independent models have been published in the ACI Materials Journal. In 1993, Gardner and Zhao produced their model which is now known as the GZ Model, ${ }^{8}$ and Gardner and Lockman recently published a new version of this model in 2001, which will be referred to as the GL Model ${ }^{9}$ in this study. In the UK, 
the creep and shrinkage design provisions in the British Standard and Eurocode 2: Design of Concrete Structures DD ENV 1992-1-1: $1992^{10}$ (EC2) are based heavily on the CEB-FIP Model Code $1990 .^{3}$

\section{Current study}

With so many different models available for the prediction of time-dependent properties, and the manner in which they do so differing between models, it is difficult to know which is best suited for a given purpose. Indeed, some are more complex than others requiring large amounts of input data such as fresh and hardened concrete properties, environmental conditions, specimen size and shape, and loading conditions.

During the past two years, a significant amount of research by the authors of this paper has focused on the time-dependent strains that develop in a range of grades of concrete, and also on current methods of predicting these strains. These studies have included comparisons with recorded data from controlled laboratory tests as well as with strains recorded in real-life structures. One of the main conclusions when comparing the predicted strains made using the prediction models listed in the Introduction with the recorded data, is that no individual model gives the closest agreement with the recorded strains in every given scenario; that is, whereas one model may prove accurate in predicting the shrinkage and creep behaviour in normal-strength concrete, the same model does not perform as well as other models when predicting the same behaviour in highstrength concrete or in real-life structures. Furthermore, whereas each of these models generally takes a different approach to shrinkage and creep prediction, the eventual magnitude of the predicted strains is generally similar for the majority of the models, despite the fact that each model focuses on different parameters depending on the model.

The question that this study aims to address is how sensitive the individual parameters are that constitute the input data for the different models, and can any one model be said to predict strains that are reasonably accurate in every situation. In this paper, the authors have used sensitivity to indicate the level of change in strain due to a prescribed change in any one of the influencing factors being considered. The authors have taken $100 \mu \varepsilon$ to be an arbitrary division between significant and marginal change in strain. In order to do this, the problem was addressed in two parts. First, the sensitivity of changes in the predicted strain to different input data was investigated at a given time, which in this case was 6 months, and second, the variation of this sensitivity over time, namely between 6 months and 2 years, was explored.

Table 1 shows a list of the models studied as well as the parameters that were considered in each model. Each of these variables was examined, and in order to do this, a range of values was adopted for use in the study as detailed in Table 2. For compressive strength, the change in the predicted strains was recorded when the 28-day compressive strength was increased from 40

Table 2. Model parameters investigated

\begin{tabular}{l|c|c}
\hline Model parameter & Control values & Alternative values \\
\hline Relative humidity: \% & 45 & 75 \\
28-day compressive strength: & 40 & 120 \\
N/mm ${ }^{2}$ & & \\
Cement content: $\mathrm{kg} / \mathrm{m}^{3}$ & 400 & 510 \\
$w / c$ ratio & $0 \cdot 56$ & $0 \cdot 24$ \\
Water content: $\mathrm{kg} / \mathrm{m}^{3}$ & 224 & 122 \\
Aggregate content: $\mathrm{kg} / \mathrm{m}^{3}$ & 1800 & 1739 \\
Slump: $\mathrm{mm}$ & 200 & 160 \\
Cement type & $\mathrm{I}$ & $\mathrm{II}, \mathrm{III}$ \\
28-day Young's modulus: & 30 & 60 \\
kN/mm ${ }^{2}$ & & \\
Curing regime & Water & Steam, sealed \\
Curing time: days & 28 & 2,7 \\
Age at loading: days & 90 & 28 \\
Size: $\mathrm{mm}$ & $600 \times 150 \times 150$ & $3000 \times 750 \times 750$ \\
$v / s$ ratio: $\mathrm{mm}$ & $37 \cdot 5$ & 187.5 \\
Shape & Prism & Cylinder, slab \\
\hline
\end{tabular}

Table 1. Parameters considered in the prediction models

\begin{tabular}{|c|c|c|c|c|c|c|c|c|c|}
\hline Description & CEB-FIP $^{3}$ & $\mathrm{EC} 2^{10}$ & $\mathrm{ACI}^{4}$ & $\mathrm{BP}-\mathrm{KX}^{5}$ & $\mathrm{BP}-\mathrm{KX}^{+}{ }^{6}$ & $\mathrm{~B} 3^{1}$ & $\mathrm{~B} 3+^{7}$ & $\mathrm{GZ}^{8}$ & $\mathrm{GL}^{9}$ \\
\hline Humidity & $\sqrt{ }$ & V & $\sqrt{ }$ & $\sqrt{ }$ & $\sqrt{ }$ & $\sqrt{ }$ & $\sqrt{ }$ & $\sqrt{ }$ & $\sqrt{ }$ \\
\hline Temperature & $\sqrt{ }$ & $\sqrt{ }$ & $\sqrt{ }$ & $\sqrt{ }$ & & V & & & \\
\hline Age at drying & & & $\sqrt{ }$ & $\sqrt{ }$ & $\sqrt{ }$ & $\sqrt{ }$ & $\sqrt{ }$ & $\sqrt{ }$ & $\sqrt{ }$ \\
\hline Age at loading & $\sqrt{ }$ & V & $\sqrt{ }$ & $\sqrt{ }$ & $\sqrt{ }$ & $\sqrt{ }$ & $\sqrt{ }$ & $\sqrt{ }$ & $\sqrt{ }$ \\
\hline Slump & & & $\sqrt{ }$ & & & & & & \\
\hline 28-day strength & $\sqrt{ }$ & $\sqrt{ }$ & $\sqrt{ }$ & $\sqrt{ }$ & $\sqrt{ }$ & $\sqrt{ }$ & $\sqrt{ }$ & $\sqrt{ }$ & $\sqrt{ }$ \\
\hline Elastic modulus & $\sqrt{ }$ & $\sqrt{ }$ & $\sqrt{ }$ & $\sqrt{ }$ & $\sqrt{ }$ & $\sqrt{ }$ & $\sqrt{ }$ & $\sqrt{ }$ & $\sqrt{ }$ \\
\hline$w / c$ & & & & $\sqrt{ }$ & V & V & $\sqrt{ }$ & & \\
\hline Cement content & & & $\sqrt{ }$ & $\sqrt{ }$ & $\sqrt{ }$ & $\sqrt{ }$ & $\sqrt{ }$ & & \\
\hline Cement type & $\sqrt{ }$ & V & $\sqrt{ }$ & V & $\sqrt{ }$ & $\sqrt{ }$ & $\sqrt{ }$ & $\sqrt{ }$ & V \\
\hline Curing regime & & & $\sqrt{ }$ & $\sqrt{ }$ & $\sqrt{ }$ & $\sqrt{ }$ & $\sqrt{ }$ & & \\
\hline Aggregate content & & & $\sqrt{ }$ & $\sqrt{ }$ & $\sqrt{ }$ & $\sqrt{ }$ & & & \\
\hline Size & $\sqrt{ }$ & $\sqrt{ }$ & $\sqrt{ }$ & $\sqrt{ }$ & $\sqrt{ }$ & $\sqrt{ }$ & $\sqrt{ }$ & $\sqrt{ }$ & $\sqrt{ }$ \\
\hline Shape & & & & $\sqrt{ }$ & & V & & & \\
\hline
\end{tabular}


to $120 \mathrm{~N} / \mathrm{mm}^{2}$. The compressive strength of concrete is dependent upon the mix proportions, in particular the water content and to a lesser degree the cement content. Many of the prediction models considered here take these factors into account and so it is not always a case of simply changing the compressive strength, but also changing the water, cement, fine and coarse aggregate contents, and even the slump. For the models that take these additional parameters into account (shown in Table 1), the mix proportions that were used were as specified by Taylor et al. ${ }^{11}$ The decision to use these two concrete strengths was due to the fact that normalstrength $40 \mathrm{~N} / \mathrm{mm}^{2}$ concrete may be considered as a typical strength for use in the construction of concrete structures, whereas with the advancement of concrete technology, the use of high-strength $120 \mathrm{~N} / \mathrm{mm}^{2}$ concrete is becoming more prevalent. Furthermore, a large amount of data was available from earlier and parallel studies, carried out in the same laboratory, using these two types of concrete. ${ }^{12-15}$

When changing the compressive strength from normal- to high-strength concrete, the stiffness of the concrete is also expected to increase. Therefore, changing the Young's modulus from 30 to $60 \mathrm{kN} / \mathrm{mm}^{2}$ accounts for a wide range of stiffness values found in modern concretes, although the compressive strength was not changed in order to assess the effect of increasing the stiffness alone.

Certain models take into account the effect of cement type and curing regime and hence the cement type was changed from type I to types II and III, while the curing regime was changed from water curing to steam curing and specimens sealed during curing (where appropriate). The curing time was varied from 2 to 7 days, and also from 7 to 28 days. The reason for this was to assess the influence of the duration of curing on the shrinkage strain. Furthermore, the time ratio from 2 to 7 days is almost identical to that from 7 to 28 days, and the change in strain over these periods could therefore be analysed in order to determine how sensitive shrinkage is to the length of curing. Following the introduction of a time element to the study, the effect that the age of concrete at loading has on the time-dependent properties was also investigated. The age at loading was chosen to be 3 months so that the concrete had sufficient time to develop the majority of its compressive strength. This is not always necessary since concrete used in construction has generally attained a desired strength level after 28 days. Therefore, the effect that loading at 28 days had on the strains developed as opposed to loading after 3 months was analysed. Obviously, in the case of shrinkage, loads are not applied to the concrete and hence it was decided to see how the shrinkage strains developed would change if shrinkage testing began in parallel with loading at these two times.

A very important influence on strain, in particular shrinkage strains due to moisture transfer with the environment, is the effect of varying relative humidity (RH). The RH parameter was therefore increased from 45 to $75 \%$, reflecting the range of humidities that a real-life structure may experience from changes in environmental conditions.

Finally, the effect that specimen geometry has on the development of strains was addressed. This involved two different factors, namely specimen size and specimen shape. Varying the specimen size affects the shrinkage strains developed because the central core of larger specimens provides a greater restraint to shrinkage, and therefore the amount of shrinkage is reduced. Creep is also reduced in larger specimens because the effects of drying creep are reduced for the same reason. In changing the size of a specimen (with each dimension changed by the same ratio), the effective thickness and volume/surface $(v / s)$ ratio are also changed. A prism of size $600 \mathrm{~mm} \times 150 \mathrm{~mm} \times 150 \mathrm{~mm}$ having a $v / s$ ratio of $37.5 \mathrm{~mm}$ was adopted as the control specimen, since laboratory work on creep has previously shown that this size of specimen yields good creep results. ${ }^{15}$ Such a specimen is typical of real-life construction, but much larger sizes are also often employed and hence it was decided to see how the predicted strains would change when the specimen size was increased by a factor of 5 ; namely a prism or column type member of size $3000 \mathrm{~mm} \times 750 \mathrm{~mm} \times 750 \mathrm{~mm}$, having a $v / s$ ratio of $187.5 \mathrm{~mm}$. Varying the specimen geometry will affect the shrinkage strains developed because specimens with longer moisture diffusion paths will have lower shrinkage rates, although size should have less effect on creep. Some models also allow the shape to be specified in the calculations; however, in changing the shape of a specimen the effective thickness and $v / s$ ratio also change, so altering the shape can effectively be incorporated into all models by changing these values. The two obvious choices for alternative shapes were cylinders and slabs, both of which are used extensively in construction. Although the shape was changed, it was important to keep the volume of concrete the same. Therefore, in the case of the cylinder, the cross-sectional area was also kept the same. However, the dimensions of the slab had to be altered significantly in order to make it precisely that, a slab. It was decided to make the slab $50 \mathrm{~mm}$ thick and hence the cross-sectional area was calculated from the volume since it had to be the same as that of the prism. It is appreciated that this is unusually thin for a slab but it was deemed sufficient for the proposed sensitivity study. A summary of each of these parameters and their values is shown in Table 2.

\section{Magnitude of change in strain}

Using the control parameters detailed in Table 2, predicted shrinkage and creep strains at 6 months for each prediction model are shown in Table 3. This study 
Table 3. Predicted shrinkage and creep strains at 6 months using control parameters

\begin{tabular}{|c|c|c|c|c|c|c|c|c|c|}
\hline Strain; $\mu \varepsilon$ & CEB-FIP ${ }^{3}$ & $\mathrm{EC} 2^{10}$ & $\mathrm{ACI}^{4}$ & $\mathrm{BP}-\mathrm{KX}^{5}$ & $\mathrm{BP}-\mathrm{KX}+{ }^{6}$ & $\mathrm{~B} 3^{1}$ & $\mathrm{~B} 3+^{7}$ & $\mathrm{GZ}^{8}$ & $\mathrm{GL}^{9}$ \\
\hline Shrinkage & $\begin{array}{r}392 \\
1181\end{array}$ & $\begin{array}{r}392 \\
1179\end{array}$ & $\begin{array}{r}589 \\
1107\end{array}$ & $\begin{array}{r}250 \\
1472\end{array}$ & 240 & 341 & $\begin{array}{r}213 \\
1105\end{array}$ & $\begin{array}{r}228 \\
1061\end{array}$ & $\begin{array}{r}549 \\
1129\end{array}$ \\
\hline
\end{tabular}

shows the differences in strain that occur due to variation of these parameters over time. Changes in predicted shrinkage strains after 6 months for the aforementioned changes in parameter values are shown in Figs 1 and 2. The parameters are split over two charts for clarity, and to distinguish between increasing and decreasing changes in strain. Notable changes in creep strain after 6 months are shown in Fig. 3. Of the other parameters which are not shown in the creep strain figure, the only major changes of note are for the curing regime parameter which when changed from water to sealed in the BP-KX and BP-KX+ models, resulted in increases of approximately $200 \mu \varepsilon$.

It is widely recognised that certain parameters have a more pronounced effect on the time-dependent properties of concrete than others. As a result, all the parameters detailed in this study were grouped according to their influence; that is, they were categorised as major or negligible. In order to ascertain how sensitive each model is when a particular parameter is varied, the effect that that variable has on the time-dependent properties was considered.

\section{Major influencing factors (at 6 months)}

These factors are those parameters which generally caused a change in strain of the order of $100 \mu \varepsilon$ or more and includes those related to environmental effects such as relative humidity, material properties such as compressive strength and stiffness, size effects such as specimen geometry, and time effects such as the age of the concrete when loaded in the case of creep.

Relative humidity. When the RH of the environment surrounding the concrete is increased, it is anticipated that shrinkage will decrease because the moisture content in the air is increased and hence, the moisture differential between the concrete and the environment is decreased. This influence is similar for creep. At some point, as the $\mathrm{RH}$ of the environment approaches $100 \%$, hygral equilibrium will be

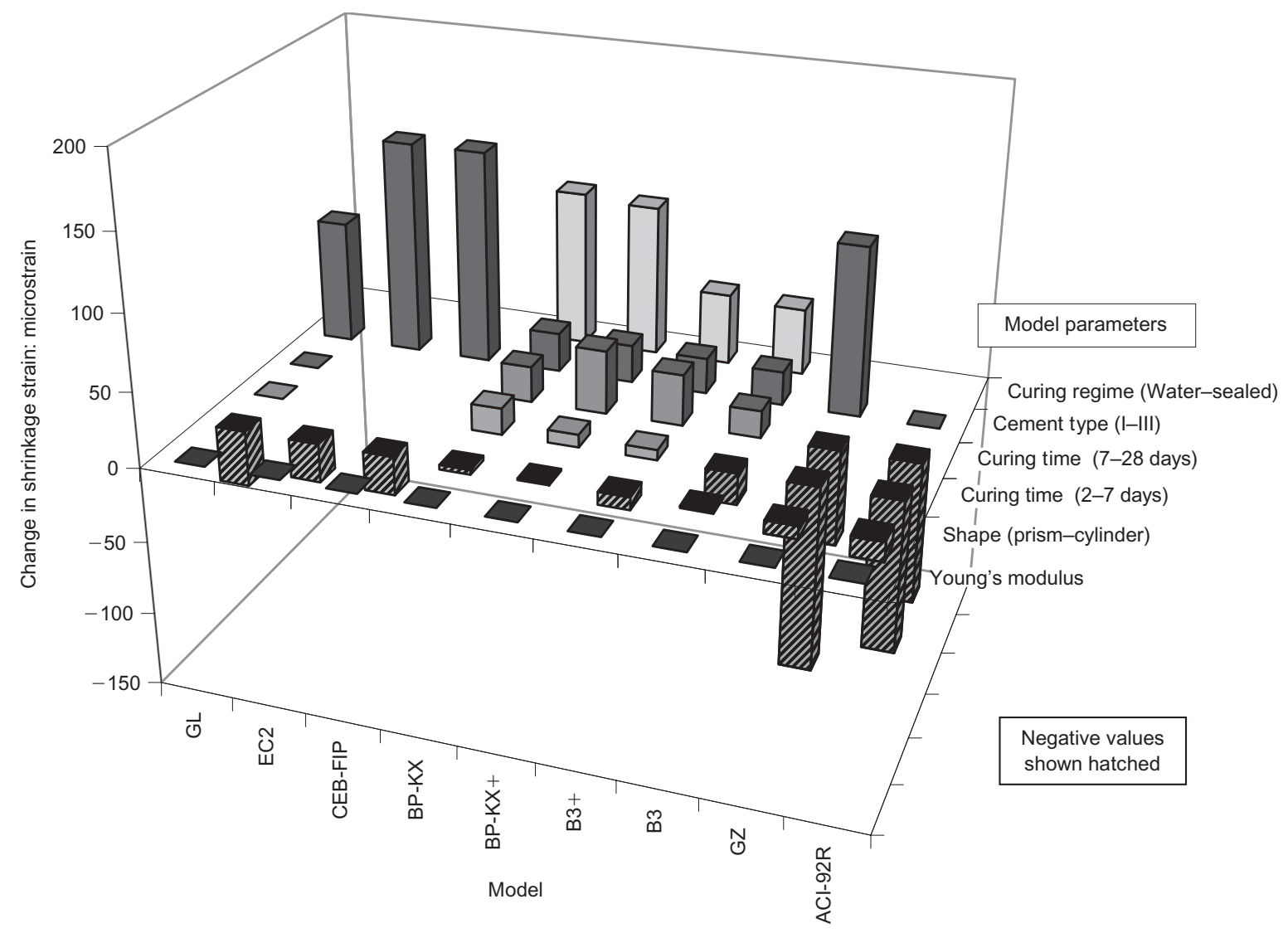

Fig. 1. Change in predicted shrinkage strain after 6 months when specific parameters are varied 


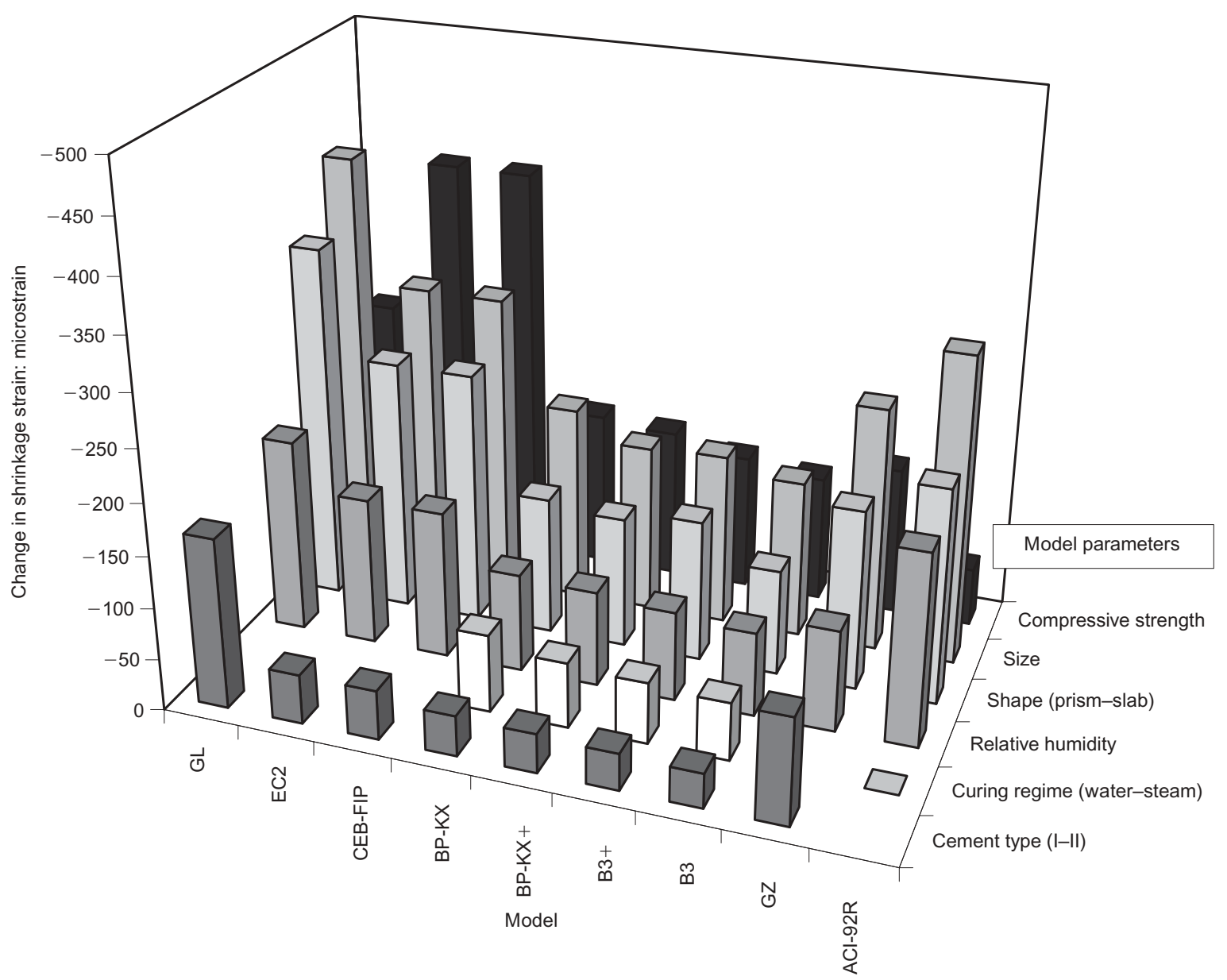

Fig. 2. Change in predicted shrinkage strain after 6 months when specific parameters are varied

reached and the shrinkage and creep strains that occur due to the effect of RH, should stop. In increasing the RH from 45 to $75 \%$ in this study, the change in shrinkage and creep strains should be significant. This was found to be the case with most of the models predicting changes in shrinkage strain in the range 80 to $190 \mu \varepsilon$ (Fig. 2) and changes in creep strain in the range 140 to $310 \mu \varepsilon$ (Fig. 3). The one exception to this was the GZ model which unexpectedly exhibited virtually no change in creep strain $(-1 \mu \varepsilon)$.

Compressive strength. When the compressive strength is increased, both shrinkage and creep are expected to decrease because in order to increase the strength of concrete the water content used in the concrete mix had to be reduced. Therefore, decreasing the amount of water in the concrete should result in less drying shrinkage and drying creep. In this study, in order to increase the strength of concrete by such a large margin from 40 to $120 \mathrm{~N} / \mathrm{mm}^{2}$, the water content had to be significantly reduced from 224 to $122 \mathrm{~kg} / \mathrm{m}^{3}$ and hence the change in shrinkage and creep strains was expected to be quite large. This was certainly the case with most models giving changes in shrinkage strains in the range 120 to $380 \mu \varepsilon$ (Fig. 2), although the ACI model predicted a smaller change of only $50 \mu \varepsilon$. The models gave differing results with regard to the change in creep strains (Fig. 3). The CEB-FIP, EC2, B3 and B3+ models exhibited changes in the range 320 to $460 \mu \varepsilon$, whereas the BP-KX and BP-KX+ models displayed changes of approximately $800 \mu \varepsilon$. The ACI model predicted a small change of approximately $50 \mu \varepsilon$ whereas the GZ and GL models suggested that there would be virtually no change whatsoever, which was unexpected. In changing strength, it is inevitable that other factors which also influence shrinkage and creep, such as the mix proportions, must also be changed. In this study, such a wide variation in predicted strains due to changes in the compressive strength was somewhat surprising, and is an indication of how difficult it is to isolate one parameter such as strength in the prediction models.

Young's modulus. Increasing the Young's modulus should have no effect on the shrinkage of concrete because it does not change the way in which water is removed from the material as was the case with all of the models. However, it should have an effect on 


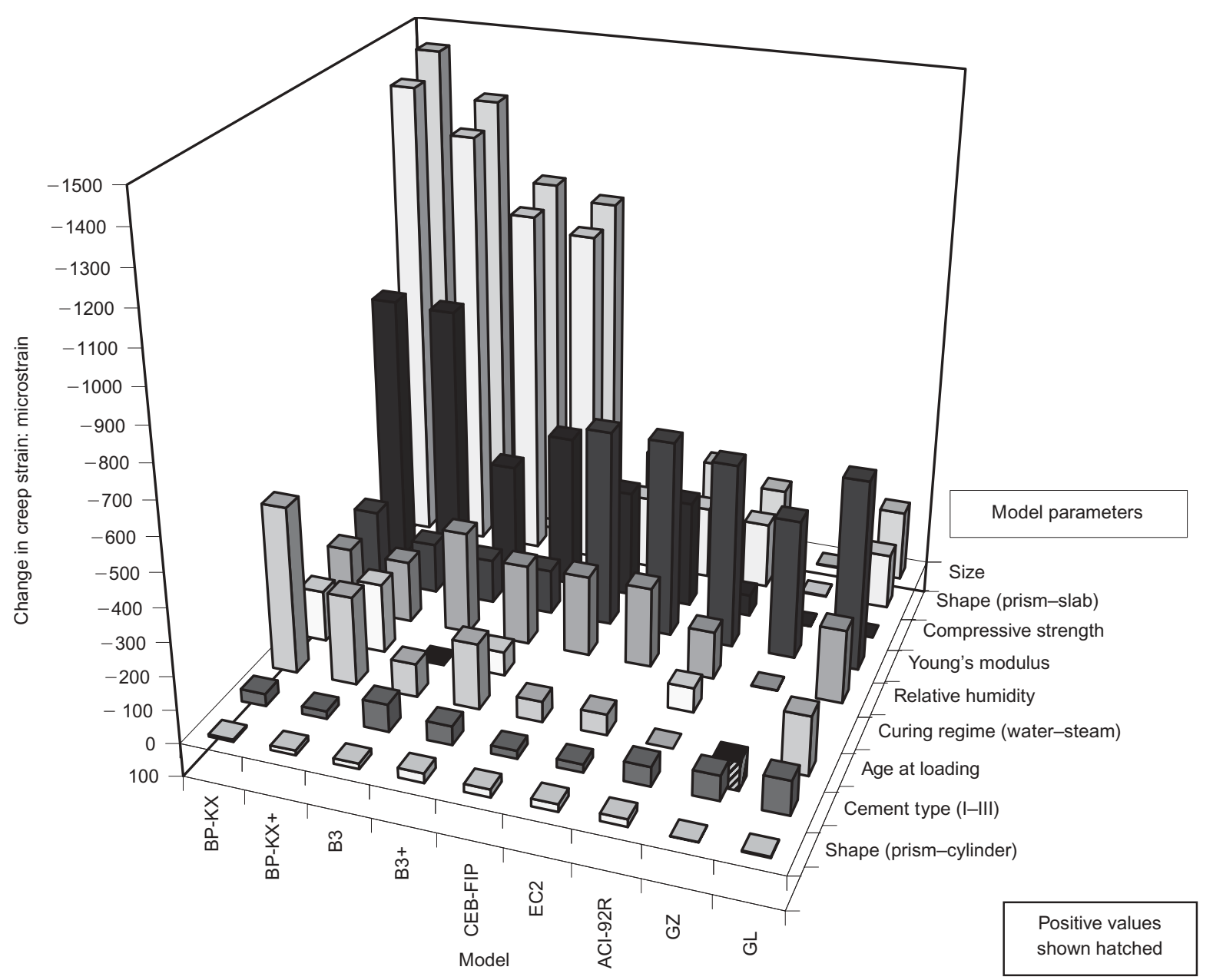

Fig. 3. Change in predicted creep strain after 6 months when specific parameters are varied

creep. Young's modulus is a measure of a material's resistance to deformation, so it can be anticipated that increasing the Young's modulus will cause an increase in the stiffness of the concrete, which means that the concrete will have a higher resistance to deformation due to the applied load and hence the creep strain should decrease. In increasing the Young's modulus from 30 to $60 \mathrm{kN} / \mathrm{mm}^{2}$, the output from the models differed by the magnitude of the change in creep strain. The $\mathrm{BP}-\mathrm{KX}, \mathrm{BP}-\mathrm{KX}+, \mathrm{B} 3$ and $\mathrm{B} 3+$ models predicted changes in creep strains in the range 130 to $220 \mu \varepsilon$, whereas the CEB-FIP, EC2, ACI, GZ and GL models exhibited more significant changes in the range 420 to $600 \mu \varepsilon$ (Fig. 3).

Size. When the size of the concrete member is increased, the volume/surface $(v / s)$ ratio increases and it might be expected that shrinkage and creep will decrease, with the effect being more pronounced in the short term. The size of a concrete specimen will influence the rate of moisture loss and the degree of overall restraint provided by the central core, which will have greater moisture content than the surface. Therefore, as the member size is increased, shrinkage should reduce owing to the fact that only the outer part of the concrete is drying and its shrinkage is restrained by the non-shrinking core. Obviously over time the core will also dry out, although this will be a gradual process and will not cause the same rapid effect witnessed in the short term. Furthermore, if no drying occurs as in very large concrete members, creep is smaller and is independent of size because there is no additional effect of drying on creep.

When the specimen size is increased from a $v / s$ ratio of 37.5 to $187.5 \mathrm{~mm}$, all models exhibited the expected decrease in shrinkage strain but differed in the magnitude by which the shrinkage strain changed (Fig. 2). The BP-KX, BP-KX+, B3 and B3+ models indicated a decrease in the range 150 to $190 \mu \varepsilon$, the CEB-FIP, EC2, ACI and GZ models suggested a decrease in the range 240 to $300 \mu \varepsilon$, whereas the GZ model predicted a decrease of approximately $400 \mu \varepsilon$.

Similarly, for the same increase in specimen size all models with the exception of the GZ model predicted the expected decrease in creep strain but differed in the magnitude by which the shrinkage strain changed (Fig. 3 ). The GZ model showed a negligible $3 \mu \varepsilon$ increase in creep strain, which was somewhat surprising. The

Magazine of Concrete Research, 2005, 57, No. 10 
CEB-FIP, EC2, ACI and GL models exhibited a decrease in the range 220 to $280 \mu \varepsilon$, whereas the $\mathrm{B} 3$ and $\mathrm{B} 3+$ models predicted a large decrease in the range 1050 to $1080 \mu \varepsilon$, and the BP-KX and BP-KX+ models exhibited an even larger decrease in the range 1442 and $1310 \mu \varepsilon$, respectively.

Shape. The effect of changing the concrete specimen shape from a prism to either a cylinder or slab varies significantly depending on the shape and the $v / s$ ratio of the specimen. Longer moisture diffusion paths lead to lower shrinkage rates; for example, a Tbeam has a high surface/volume ratio and will therefore dry and consequently shrink more rapidly than a beam with a square cross-section of the same area. The same principle applies with drying creep. Therefore, since the cylinder had the same cross-sectional area as the prism, the $v / s$ ratio was also very similar and there should be very little difference in the shrinkage and creep strains between these two shapes, with the cylinder possibly having lower shrinkage strains due to the geometry of the cross-section. However, the effect of changing the shape from a prism to a slab of the same volume of concrete should have been much greater as, although the depth of the specimen was decreased from 600 to $50 \mathrm{~mm}$, the cross-sectional area was increased from 22500 to $270000 \mathrm{~mm}^{2}$, and therefore the $v / s$ ratio was increased from 37.5 to $129.9 \mathrm{~mm}$. The minimum moisture diffusion path length in the prism was $75 \mathrm{~mm}$ whereas in the slab the minimum length was $25 \mathrm{~mm}$. Therefore, shrinkage and drying creep should occur far more rapidly with much lower strains, and the difference in strain should be large.

When the shape is changed from a prism to a cylinder (Fig. 1), all models exhibited either the expected negligible decreases or unexpected but negligible increases in shrinkage strain of up to $25 \mu \varepsilon$. All models indicated the expected negligible decreases in creep strain of up to $30 \mu \varepsilon$. When the shape is changed from a prism to a slab the changes in the time-dependent strains were much larger (Fig. 2). All models exhibited an expected decrease in shrinkage strain of 100 to $343 \mu \varepsilon$. Similarly, all models showed the expected decrease in creep strain but differed in the magnitude by which the creep strain changed (Fig. 3). The GZ model indicated a negligible $3 \mu \varepsilon$ decrease in creep strain that was somewhat surprising, the CEB-FIP, EC2, ACI and GL models suggested a decrease in the range 160 to $220 \mu \varepsilon$, whereas the RILEM models indicated a surprisingly large decrease in the range 1000 to $1384 \mu \varepsilon$.

Age at loading. The effect that changing the age at which the concrete is loaded has on the timedependent deformations of concrete applies to the creep strains only, since the application of load only affects the magnitude of creep. If concrete is loaded after 3 months as opposed to 28 days, it was ex- pected that the magnitude of the creep strains should be less as the concrete will have hydrated for longer, so allowing more $\mathrm{C}-\mathrm{S}-\mathrm{H}$ to develop, and strength and stiffness to increase so providing a greater resistance to deformation. Therefore, the magnitude of the change in creep strain should be greater in the short term for the concrete loaded at 28 days, as it is still gaining strength, as opposed to the concrete loaded at 3 months, which will have gained the majority of its strength.

This is a slightly unfair comparison as the concrete will be a different age depending on when it was loaded. The ACI and GZ models indicated an increase in creep strain with loading ages of 5 and $91 \mu \varepsilon$, respectively, which is contrary to what was expected. All other models gave the expected decrease in creep strain, but the magnitude of the change in strain differed (Fig. 3). The CEB-FIP, EC2 and B3 models all showed deceases in strain in the range 80 to $100 \mu \varepsilon$, whereas the BP-KX, BP-KX+, B3+ and GL models suggested deceases in strain in the range 200 to $270 \mu \varepsilon$.

\section{Negligible influencing factors (at 6 months)}

These factors are those parameters which generally cause a change in strain of the order of approximately $100 \mu \varepsilon$ or less and include those related to material composition such as cement type, and those related to the hydration process such as the curing regime and duration. This range of strain variation $(0$ to $100 \mu \varepsilon)$ is arbitrary and is open to debate.

Cement type. It was anticipated that the effect of changing the cement type from Ordinary Portland Cement (OPC) (Type I) to either slowly hardening cement (Type II) or rapid-hardening cement (Type III) would be more pronounced within the first 3 months. With slowly hardening cement, the rate of gain in compressive strength of the concrete will be slower than in OPC, whereas with rapid-hardening cement, the rate of gain in compressive strength of the concrete will be much quicker. However, after 6 months most of the compressive strength of the concrete will have developed for each of the cement types and the differences in shrinkage and creep strains should be minimal. If there were any differences at this time, there should be an increase in shrinkage and creep strains when the cement type is changed from OPC to rapid hardening and a decrease when it is changed from OPC to slowly hardening.

When the cement type was changed from OPC to slowly hardening cement (Fig. 2), all models which considered this parameter exhibited the expected decrease in shrinkage strain in the range 50 to $70 \mu \varepsilon$, with the exception of the GZ and GL models which suggested changes of 120 and $220 \mu \varepsilon$, respectively. The same models showed either the expected increase or an unexpected decrease in creep strain in the range 30 to 
$80 \mu \varepsilon$. When the cement type was changed from OPC to rapid hardening (Fig. 1), all relevant models indicated an expected increase in shrinkage strain in the range 25 to $140 \mu \varepsilon$, whereas the ACI model showed no change. However, all models suggested an unexpected decrease in creep strain in the range 25 to $100 \mu \varepsilon$ (Fig. 3).

Curing regime. In a similar fashion to the effect of changing the cement type, the effect of changing the curing regime from water curing to steam curing or sealing the concrete during curing is more pronounced in the short term. When concrete is cured in steam, the curing temperature is increased and the development of calcium silicate hydrate $(\mathrm{C}-\mathrm{S}-\mathrm{H})$ gel within the cement paste during hydration becomes more rapid, so increasing the rate of strength gain and the stiffness of the concrete. Since hydration occurs at a quicker rate, it finishes earlier and hence there is less water held in the concrete, and therefore shrinkage and drying creep is reduced. However, after 6 months most of the compressive strength of the concrete will have developed and the differences in shrinkage and creep strains should be minimal. When concrete is sealed during curing, the concrete is denied water, hydration comes to a halt sooner and so there should be a noticeable increase in shrinkage over the early life of the concrete. Similarly, since hydration comes to a halt, the development of the concrete strength is reduced and since the compressive strength is reduced, the creep strain should increase.

When the curing regime was changed from water to either steam or sealed curing, all the models in which this parameter could be varied exhibited negligible changes in shrinkage (Figs 1 and 2, respectively) and creep strains (Fig. 3) as expected, with the exception of the BP-KX and BP-KX+ models which predicted unexpected and surprisingly large decreases in creep strain in the range 150 to $210 \mu \varepsilon$.

Duration of curing. It is anticipated that in the short term, the effect that increasing the curing time has on the time-dependent deformation of concrete will become more noticeable the longer the period of initial curing. When concrete is placed under a controlled curing regime, as the curing time increases more cement becomes hydrated and the volume of unhydrated cement particles reduces. Obviously, the longer the concrete is cured, the more $\mathrm{C}-\mathrm{S}-\mathrm{H}$ is developed within the paste and the greater the strength and stiffness of the concrete that results. Curing concrete for 2 days would mean that the concrete is still very weak and therefore the creep strain would be high because of this. After 7 days, the concrete would be well cured and adequate strength and stiffness would have developed, so the creep strain should be less. Similarly, after 28 days the concrete will have cured further, the strength and stiffness of the concrete will be larger still and the creep strain will further reduce. However, since hydration is most rapid between 2 and 7 days, then the change in shrinkage and creep strains between 7 and 28 days is expected to be only marginally larger than the corresponding change in strain between 2 and 7 days.

When the curing time was changed from 2 to 7 days and from 7 to 28 days, all models showed a negligible change in shrinkage strain with the exception of the ACI and GZ models which, for the former, exhibited decreases of 100 and $130 \mu \varepsilon \varepsilon$ (Fig. 1). Likewise, all models showed negligible changes in creep strain indicating that what is important is the percentage change in time rather than the actual duration when predicting strains.

\section{Magnitude of change in strain over time}

It is also important to see how the predicted strains change over time when these parameters are varied. Notable changes in shrinkage strain between 6 months and 2 years are shown in Fig. 4. Of the other parameters investigated, the only major changes in shrinkage strain of note were for the curing regime parameter when changed from water to sealed, for which the BP$\mathrm{KX}$ and BP-KX+ models showed increases of approximately $200 \mu \varepsilon$, and also for the cement type parameter when changed from type I to type III, for which the CEB-FIP, EC2, GZ and GL models indicated increases in the range 100 to $230 \mu \varepsilon$.

Notable changes in creep strain between 6 months and 2 years are shown in Fig. 5. Of the other parameters investigated, the only major changes in creep strain of note were also for the curing regime parameter when changed from water to sealed, for which the BP$\mathrm{KX}$ and $\mathrm{BP}-\mathrm{KX}+$ models showed increases of approximately $300 \mu \varepsilon$. As can be seen, certain parameters resulted in increases in strain from 6 months to 2 years whereas some parameters resulted in a decrease. Tables 4 and 5 show the magnitude of the changes and also the percentage change (in brackets) in shrinkage and creep strains over this period. It is important to note that positive values of the percentage change showed an increase in the magnitude of the change in strain whereas negative values showed a decrease. It is also important to look at the magnitude of the change in strain as well as the percentage change because whereas the percentage change in strain was very large in some cases (much greater than $100 \%$ ), the actual change in the magnitude of strain was often very small (less than $50 \mu \varepsilon)$. The effect that changing these parameters has on the predicted strains over time is discussed in the following section.

Magazine of Concrete Research, 2005, 57, No. 10 


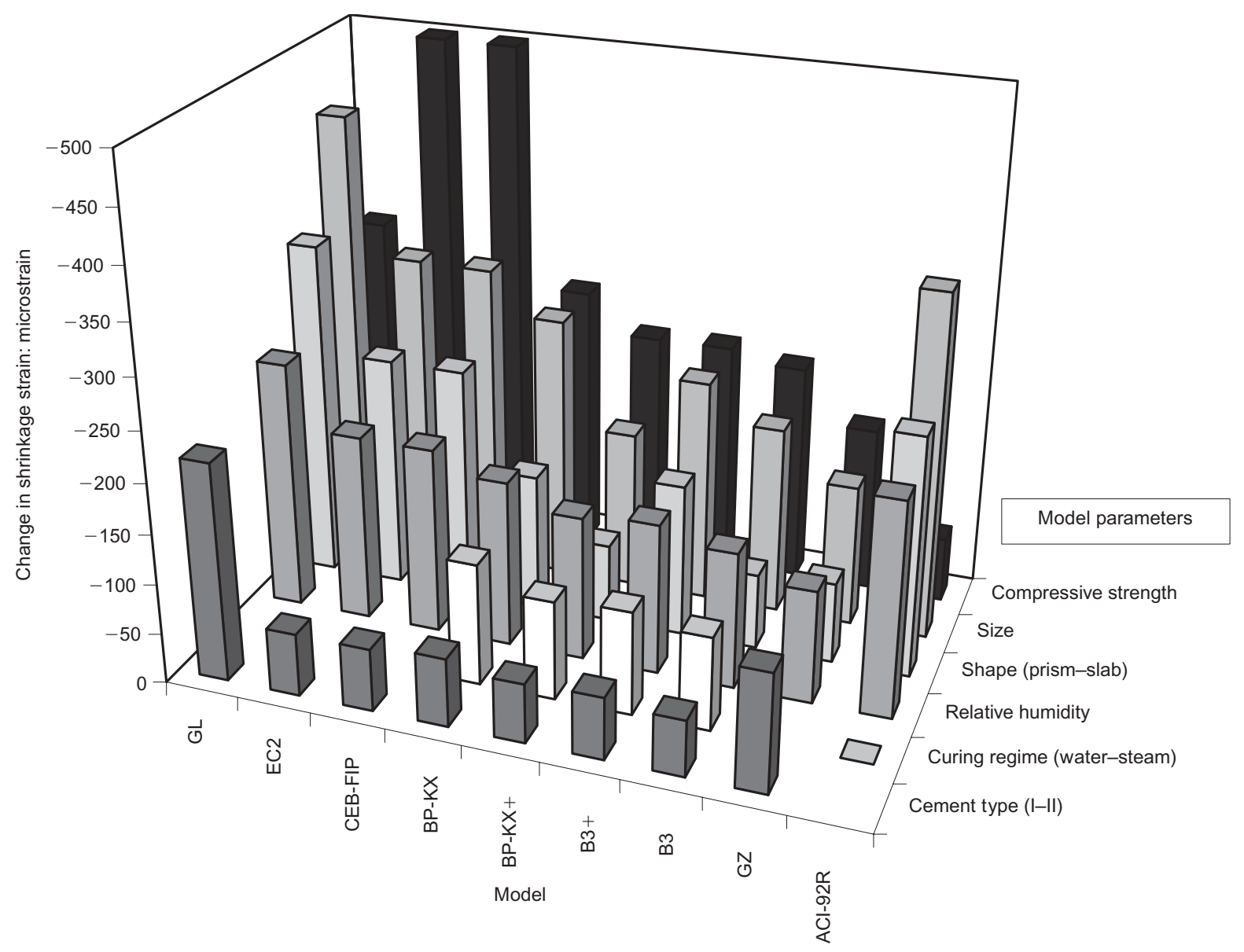

Fig. 4. Change in predicted shrinkage strain after 2 years when specific parameters are varied

\section{Major influencing factors over time}

These are factors that still had a pronounced effect on the time-dependent properties of concrete after 6 months and 2 years, such as relative humidity, compressive strength, stiffness and specimen geometry.

Relative humidity. It has already been noted that the relative humidity of the environment surrounding the concrete might be expected to have a marked effect on the time-dependent strains in concrete. The effect should still be noticeable in the change in shrinkage strain after 6 months, but the change in creep strain over the same period due to the influence of drying creep can be expected to be even greater. All models (with the exception of the ACI and GZ models) were found to be moderately sensitive to changes in RH over time (Table 4), predicting changes in shrinkage strain in the range 40 to $70 \mu \varepsilon$, whereas the ACI model predicted a smaller change of $28 \mu \varepsilon$ and the GZ model showed no change, which was somewhat surprising. Similarly, all models (with the exception of the ACI, GZ and B3 models) suggested the same noticeable change in creep strain (Table 5) in the range 60 to $130 \mu \varepsilon$, whereas the B3 model indicated a large change of $261 \mu \varepsilon$ with a percentage change over time of $84 \%$. The ACI model predicted a small change of $32 \mu \varepsilon$, and the GZ model showed no change in creep strain, which was again unexpected.

Compressive strength. The effect that changing the compressive strength of the concrete has on the time-dependent deformations should also be significant due to the fact that increasing the compressive strength means reducing the water content causing the majority of both creep and shrinkage to occur far earlier in the life of the concrete. All models (with the exception of the ACI and GZ models) demonstrated that this was the case, predicting changes in shrinkage strain in the range 75 to $120 \mu \varepsilon$ (Table 4), while the ACI and GZ models predicted much smaller changes in the range 9 to $22 \mu \varepsilon$, which were somewhat smaller than those anticipated. Similarly for creep, the ACI model predicted a negligible change in creep strain between 6 months and 2 years of $15 \mu \varepsilon$ whereas the GZ and GL model showed no change in creep strain (Table 5), which was again surprising. The CEB-FIP and EC2 models, which incidentally have shown almost identical shrinkage and creep strains throughout this study due to the fact that they are based on similar prediction formulae, 


\begin{tabular}{|c|c|c|}
\hline & 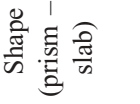 & 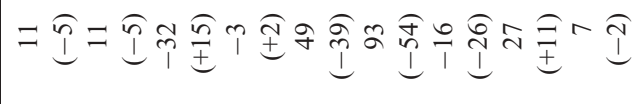 \\
\hline & 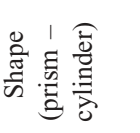 & 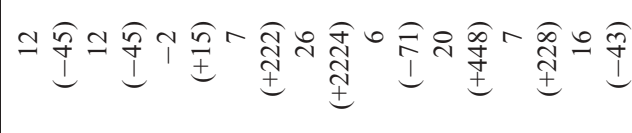 \\
\hline & $\stackrel{\mathscr{N}}{\tilde{n}}$ & 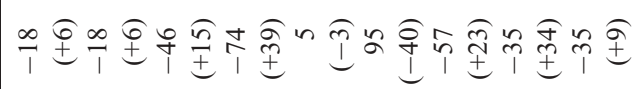 \\
\hline & 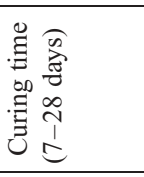 & 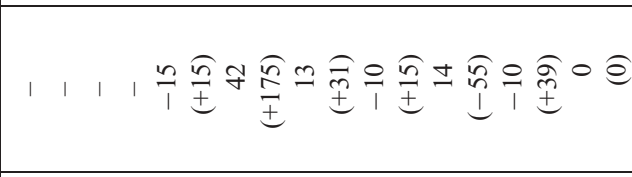 \\
\hline & 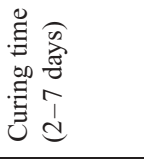 & 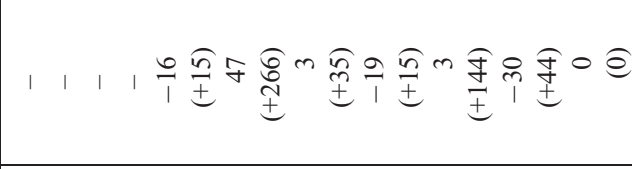 \\
\hline & 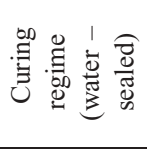 & 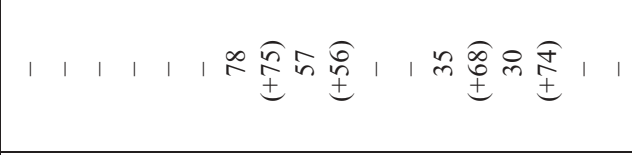 \\
\hline & 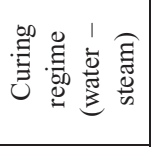 & | 1 1 , o \\
\hline & 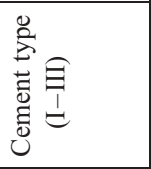 & 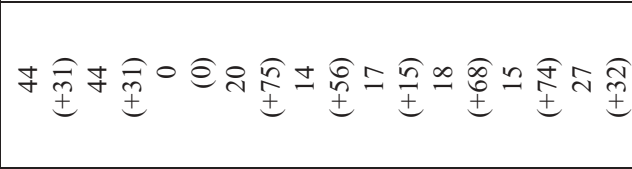 \\
\hline & 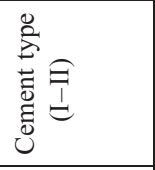 & 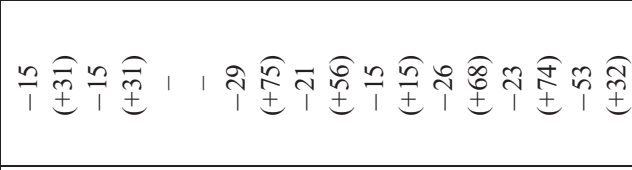 \\
\hline & 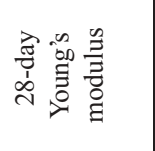 & - \\
\hline & 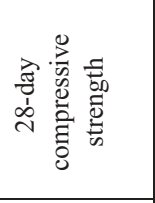 & 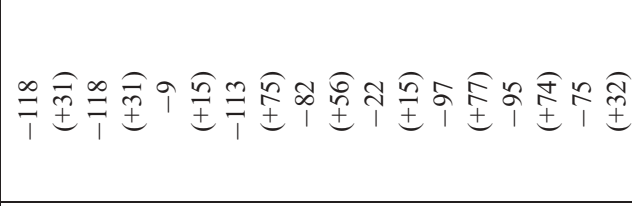 \\
\hline & 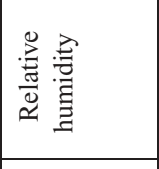 & 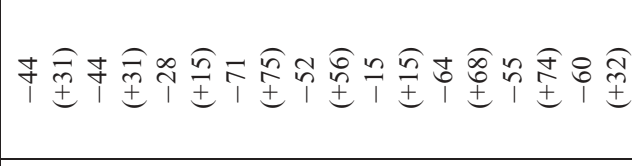 \\
\hline & $\frac{\bar{s}}{0}$ & 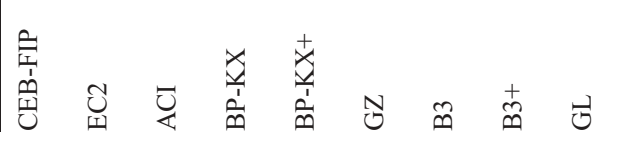 \\
\hline
\end{tabular}




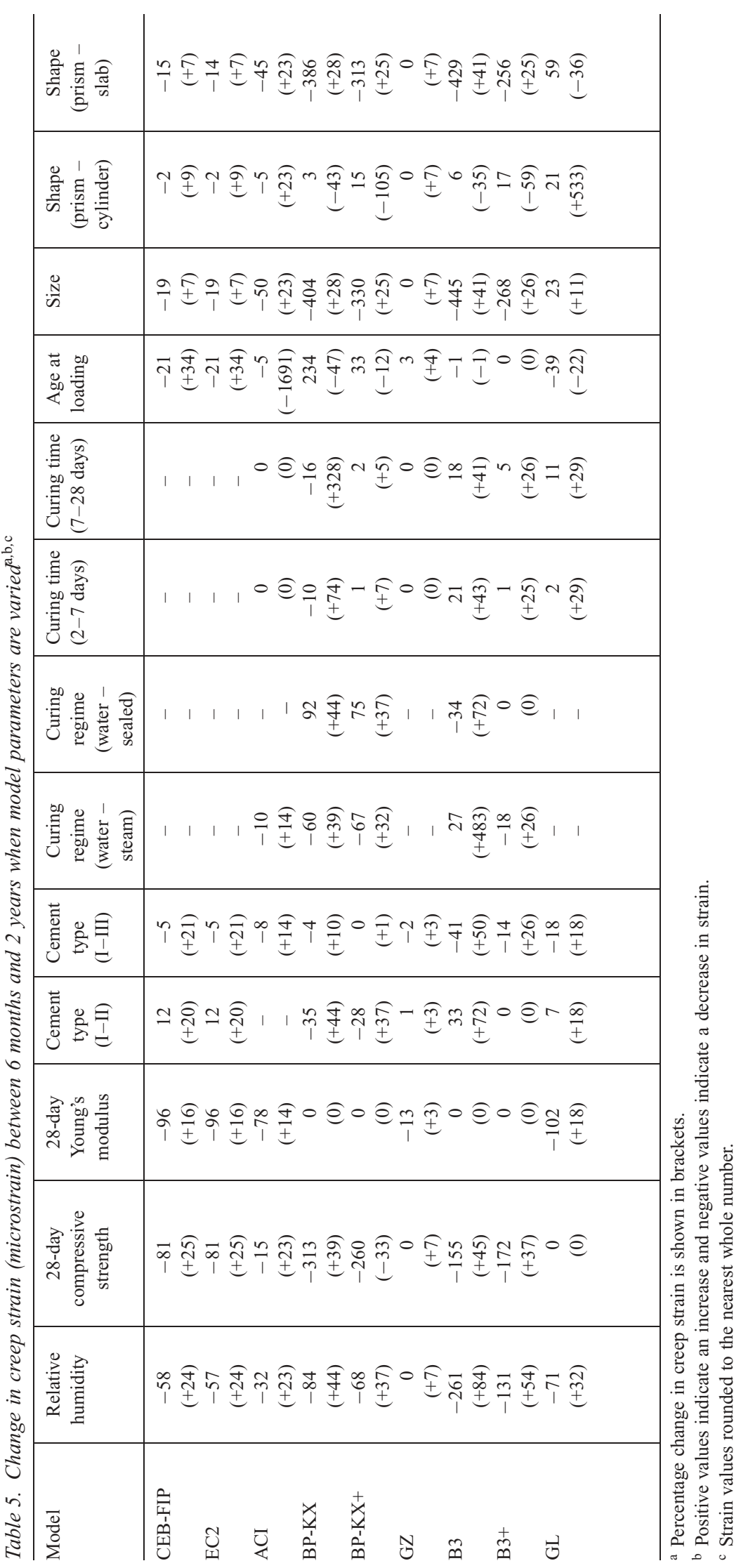




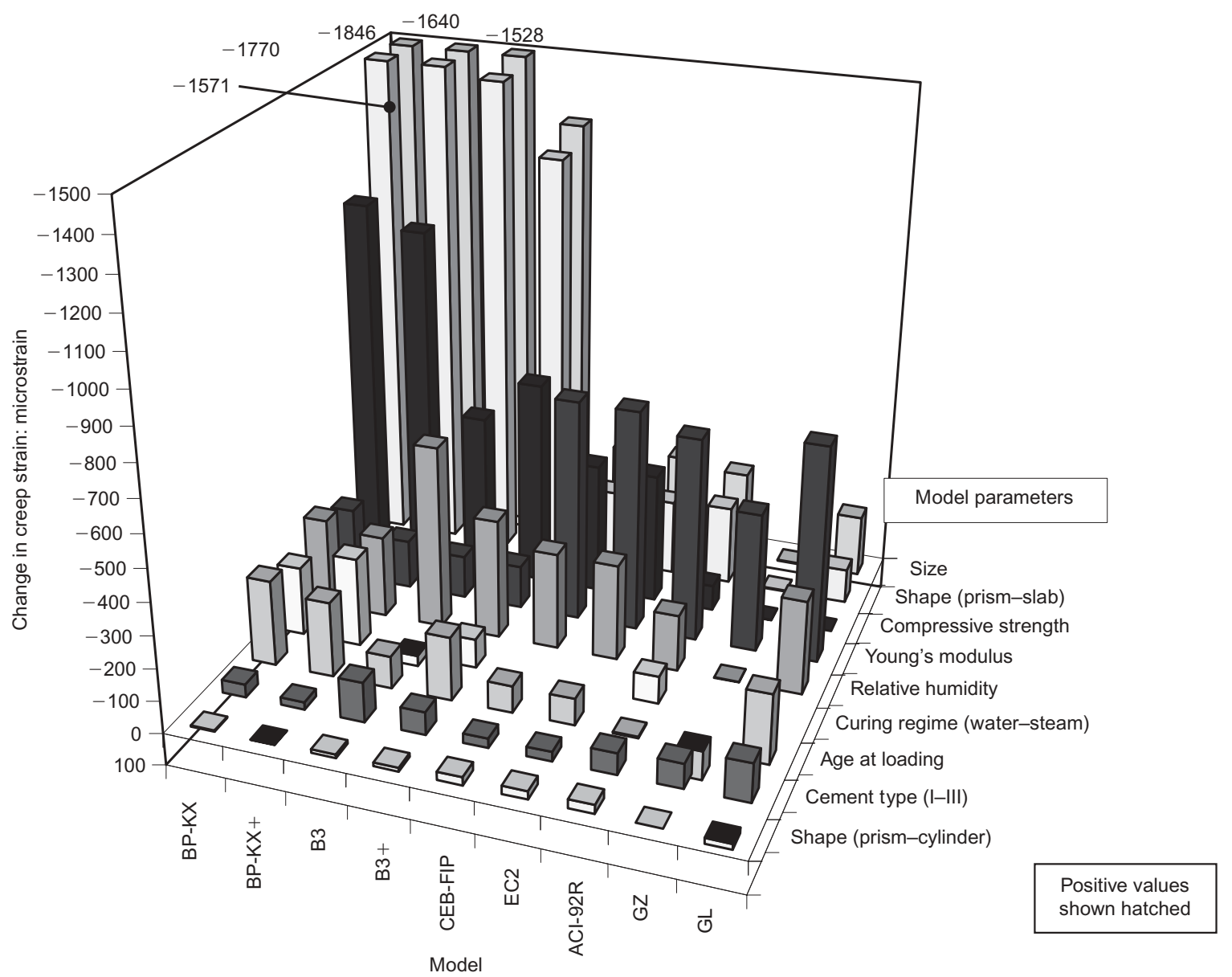

Fig. 5. Change in predicted creep strain after 2 years when specific parameters are varied.

indicated a change in creep strain of $81 \mu \varepsilon$, whereeas the B3 and B3+ models exhibited larger changes in the range 150 to $170 \mu \varepsilon$, and the $\mathrm{BP}-\mathrm{KX}$ and $\mathrm{BP}-$ $\mathrm{KX}+$ models exhibited changes of 260 and $310 \mu \varepsilon$. Percentage changes in creep strain were also relatively small for all models (less than 45\%) indicating that the sensitivity to change over time is relatively low.

Young's modulus. As previously mentioned, changing the Young's modulus of the concrete should have no effect on the shrinkage strain and this was again the case for all models. Increasing the Young's modulus should only have an effect at the time of load application when the instantaneous strain developed. Between the age of 6 months and 2 years, it is expected that there will be no further effect on the creep strain by changing this parameter, and the change in strain should be negligible. This was certainly the case for the $\mathrm{BP}-\mathrm{KX}, \mathrm{BP}-\mathrm{KX}+, \mathrm{B} 3$ and $\mathrm{B} 3+$ models, in which there was no change at all, whereas the GZ model exhibited a negligible change of $13 \mu \varepsilon$ (Table 5). However, the CEB-FIP, EC2, ACI and GL models showed changes in creep strain in the range 80 to $100 \mu \varepsilon$, which was unexpected.

Size. The effect that changing the size of the specimen has on the predicted strains over time was expected to be very similar to changing the specimen shape from a prism to a slab since the $v / s$ ratio is similar. There should be a marginal change in the shrinkage strain and this was the case with all models showing changes in shrinkage strain of less than $100 \mu \varepsilon$ (Table 4). Again, it might have been expected that there would be a noticeable change in creep strain over time due to the fact that drying creep should be still occurring in the prism whereas it should have finished in the slab due to the larger $v / s$ ratio and shorter moisture diffusion paths. The models predicted conflicting results when this change in creep strain over time is observed (Table 5). The CEB-FIP, EC2, ACI and GL models all showed small, negligible changes in creep strain of up to $50 \mu \varepsilon$, whereas the $\mathrm{BP}-\mathrm{KX}, \mathrm{BP}-\mathrm{KX}+, \mathrm{B} 3$ and $\mathrm{B} 3+$ models showed much larger changes in creep strain in the range 270 to $450 \mu \varepsilon$. The GZ model again exhibited no change which was unexpected. Percentage changes 
in creep strain were also relatively small for all models indicating that the sensitivity to change over time is low.

Shape. When the specimen shape is changed from a prism to a cylinder, all models demonstrated a negligible change in shrinkage and creep strains (Tables 4 and 5, respectively), which was as expected since the cross-sectional area and $v / s$ ratio were very similar for the two different shapes. As the majority of shrinkage will have taken place in the first 6 months, there is little change in shrinkage strain expected even when the specimen shape is changed from a prism to a slab. This was certainly the case with regard to the change in shrinkage strain with all models showing negligible changes in strain. However, since the majority of drying creep will have taken place within the slab over the first 6 months, a noticeable change in creep strain was expected when the specimen shape was changed from a slab to a prism as drying creep will still be occurring in the prism after this time due to the smaller $v / s$ ratio. The models indicated conflicting results when this change in creep strain over time was observed (Table 5). The CEB-FIP, EC2, ACI, GZ and GL models all depicted negligible changes in creep strain of up to $60 \mu \varepsilon$, which was unexpected, whereas the BP-KX, BP-KX+, $\mathrm{B} 3$ and $\mathrm{B} 3+$ models predicted much larger changes in creep strain in the range 250 to $430 \mu \varepsilon$.

\section{Negligible influencing factors over time}

As previously mentioned, the effect of changing certain parameters will have a more pronounced effect in the early life of the concrete when hydration is still occurring and the rate in gain of the compressive strength is at its most rapid. However, in the long term (6 months and onwards), the effect of these parameters on the shrinkage and creep of concrete should be minimal. These parameters include the cement type, length of curing and the curing regime, and the shape of the concrete specimen. Percentage changes in creep and shrinkage strain for the following parameters were often large, but because the magnitude of change was relatively small, it can be concluded that all models gave an adequate representation of the change in shrinkage and creep strains over time when the parameter in question was changed.

Cement type. When the cement type was changed from OPC to either slowly hardening or rapid hardening, all models exhibited negligible changes in shrinkage and creep strain of less than $50 \mu \varepsilon$ (Tables 4 and 5 , respectively).

Curing regime. The CEB-FIP, EC2, GZ and GL models do not consider this parameter when predicting shrinkage and creep strains. When the curing regime was changed from water to steam, all other models showed a negligible change in shrinkage strain (Table 4). Similarly, when the curing regime was changed from water to sealed all models again exhibited negligible changes for both shrinkage and creep, which was as expected.

Duration of curing. Since the majority of curing will have taken place prior to 6 months regardless of how long the concrete was cured initially, it is anticipated that there will be little change in strain from 6 months to 2 years. All models showed the expected negligible change in shrinkage and creep.

Age at loading. As previously mentioned the age at loading applies to the creep strains only (Table 5). After 2 years, whether the concrete was loaded after 28 days or 3 months was expected to have little effect on the magnitude of the creep strain since hydration and the majority of strength development will have come to an end. However, it is probable that after 6 months there would still be some effect on the magnitude of creep strain and so there may be a small difference in the strain values. All models with the exception of the BP-KX model showed a negligible change in strain of less than $40 \mu \varepsilon$, whereas the BP-KX model suggested an exceptionally large change in creep strain of $234 \mu \varepsilon$, which was somewhat surprising.

\section{Conclusions}

From this study of the CEB-FIP, EC2, ACI, BP-KX, $\mathrm{BP}-\mathrm{KX}+, \mathrm{GZ}, \mathrm{B} 3, \mathrm{~B} 3+$, and GL prediction models, it has been concluded that certain parameters have a much bigger influence on the time-dependent strains than others. In order to carry out this study, significant variation was ascribed to a number of parameters. The authors are aware that in some instances, the parameters used went beyond that recommended by the authors of the various models. Nevertheless, it was considered essential to test the various models at these extremes in order to appreciate the full impact of possible variations in the various parameters. It has been confirmed that the two most influential parameters on the shrinkage and creep of concrete are the relative humidity of the environment and the compressive strength of concrete, although the Young's modulus of concrete has an effect on the creep strain, albeit to a lesser extent. An appreciation of these factors and the sensitivity level of these parameters as opposed to the less significant factors must therefore be taken into account when selecting a prediction model for the time-dependent analysis of concrete materials and structures.

It has been determined that each of these models is more sensitive to some parameters than others, with the parameters that are the most sensitive being dependent 
upon the model. However, if the strains predicted by each model are even remotely similar over time then this would suggest that for any given model, any parameters that do not appear to indicate entirely accurate behaviour when changed may well counterbalance each other, and any errors are therefore cancelled out. This would suggest that no one model can be said to be more accurate, and when deciding which model to use when predicting shrinkage and creep strains, it is prudent to look at the individual parameters on which each model is dependent, and assess the sensitivity level of each of these parameters so that the most appropriate model for the specific circumstances can be selected.

The assumptions made in this study are a matter for debate, and therefore a parallel laboratory study using these creep and shrinkage models is currently underway. The aim of this study is to compare the experimental results obtained with the findings of the work reported here and to assess the accuracy of these assumptions and the analysis herein.

Finally, the study has shown that the CEB-FIP Model Code 1990 and the Eurocode 21992 have predicted virtually identical strains for both creep and shrinkage for all varied parameters. This is hardly surprising since the two models share exactly the same shrinkage formulae, and very similar creep formulae.

\section{Acknowledgements}

The first author wishes to express thanks to Carillion, for partial support to carry out the work reported in this study.

\section{References}

1. BAŽANT Z. P. and BAwEJA S. Creep and shrinkage prediction model for analysis and design of concrete structures - Model B3. Published as a draft RILEM recommendation in Materials and Structures, 1995, 28, No. 180, 357-365, No. 181, 415-430, No. 182, 488-495, with errata in 1996, 29, No. 186, 126.

2. Ross A. D. Concrete creep data. The Structural Engineer 1937, 15, No. 8, 314-326.

3. Comité Euro-International du Béton - FÉdération In- ternationale de la Précontraine (CEB-FIP) CB-FIP Model Code Design Code. Thomas Telford, London, 1990.

4. ACI Committee 209. Prediction of Creep, Shrinkage and Temperature Effects in Concrete Structures (ACI 209R-92). American Concrete Institute, Detroit, MI, 1992.

5. BaŽAnt Z. P., KIM J. K. and PANUla L. Improved prediction model for time dependent deformations of concrete. Part 1 shrinkage. Materials and Structures, 1991, 24, No. 143, $327-$ 345; Part 2 - Basic creep. Materials and Structures, 1991, 24, No. 144, 409-420; Part 3 - Creep at drying. Materials and Structures, 1992, 25, No. 145, 21-28; Part 4 - Temperature effects. Materials and Structures, 1992, 25, No. 146, 84-94; Part 5 - Cyclic load and cyclic humidity. Materials and Structures, 1992, 25, No. 147, 163-169.

6. BaŽAnt Z. P., XI Y. and BaWEJA S. Improved prediction model for time dependent deformations of concrete, Part 7 - Short form of BP-KX model, statistics and extrapolation of short-time data. Materials and Structures, 1993, 26, No. 164, 567-574.

7. BAŽAnT Z. P. and BAwEJA S. Short form of creep and shrinkage prediction model B3 for structures of medium sensitivity. Materials and Structures, 1996, 29, No. 194, 587-593.

8. Gardner N. J. and ZhaO J. W. Creep and shrinkage revisited. ACI Materials Journal, 1993, 90, No. 3, 236-246.

9. Gardner N. J. and Lockman M. J. Design provisions for drying shrinkage and creep of normal-strength concrete. ACI Materials Journal, 2001, 98, No. 2, 159-167.

10. British Standards Institution. Eurocode 2: Design of Concrete Structures - Part 1: General Rules and Rules for Buildings. DD ENV 1992-1-1: 1993. British Standards Institution, Milton Keynes, 1992.

11. TAYlor M. R., Lydon F. D. and BARR B. I. G. Mix proportions for high strength concrete. Construction and Building Materials, 1996, 10, No. 6, 445-450.

12. Conroy-Jones G. A. and Barr B. I. G. Effect of curing on the tensile strength of medium to high strength concrete. Magazine of Concrete Research, 2004, 56, No. 3, 151-158.

13. Barr B. I. G. and El-Baden A. S. A. Shrinkage properties of normal and high strength fibre reinforced concrete. Proceedings of the Institution of Civil Engineers-Structures and Buildings 2003, 156, No. 1, 15-25.

14. Barr B. I. G., Howells R. W. and Lark R. J. A characterisation of the serviceability behaviour of post-tensioned concrete structures. Proceedings of the $3^{\text {rd }}$ International Conference on Current and Future Trends in Bridge Design, Construction and Maintenance, Shanghai, 2003, pp. 207-216.

15. Howells R. W. Creep of High Strength Concrete. Undergraduate Final Year Project, submitted to the Division of Civil Engineering, School of Engineering, University of Wales, Cardiff, 2001.

Discussion contributions on this paper should reach the editor by 1 June 2006 